\title{
Chaotic Breathers in Delayed Electro-Optical Systems
}

\author{
Y. Chembo Kouomou, ${ }^{1, *}$ Pere Colet, ${ }^{1}$ Laurent Larger, ${ }^{2}$ and Nicolas Gastaud ${ }^{3}$ \\ ${ }^{1}$ Instituto Mediterráneo de Estudios Avanzados IMEDEA (CSIC-UIB), Campus Universitat de les Illes Balears, \\ E-07122 Palma de Mallorca, Spain \\ ${ }^{2}$ UMR CNRS FEMTO-ST 6174/Optics Department, Université de Franche-Comté, 16 Route du Gray, 25030 Besançon cedex, France \\ ${ }^{3}$ GTL-CNRS Telecom, UMR FEMTO-ST 6174, 2-3 rue Marconi, 57070 Metz cedex, France
}

(Received 24 September 2004; published 9 November 2005)

\begin{abstract}
We show that in integro-differential delayed dynamical systems, a hybrid state of simultaneous fastscale chaos and slow-scale periodicity can emerge subsequently to a sequence of Hopf bifurcations. The resulting time trace thereby consists in chaotic oscillations "breathing" periodically at a significantly lower frequency. Experimental evidence of this type of dynamics in delayed dynamical systems is achieved with a Mach-Zehnder modulator optically fed by a semiconductor laser and is subjected to a delayed nonlinear electro-optical feedback. We also propose a theoretical understanding of the phenomenon.
\end{abstract}

DOI: 10.1103/PhysRevLett.95.203903

PACS numbers: 42.65.Sf, 05.45.Jn

Since the pioneering work of Ikeda [1], important experimental and theoretical research has focused on the dynamics of nonlinear optical systems with delayed feedback, and the so-called Ikeda equation has been converted into a paradigm for the study of differential-delay systems in physics. In particular, a wide variety of acousto-optical and electro-optical systems can be modeled with an Ikeda equation $[2,3]$, or one of its modified versions. For example, some acousto-optical devices were modeled with an Ikeda-like equation containing higher-order differential terms [4], while in another setup, the limit case of a zero delay was studied [5]. In all cases, several types of instabilities and nonlinear behaviors have been evidenced and investigated, from bistability to hyperchaos. However, if a wide variety of differential delayed systems have been investigated, only a very limited interest has been paid until now to the nonlinear dynamics and bifurcation behavior of integro-differential delayed systems. Here, a quite interesting and unreferenced phenomenon can be observed: simultaneous fast-scale chaos and slow-scale periodicity.

In this Letter, the system under study corresponds to the experimental setup of Fig. 1. The electro-optical feedback loop is composed with several elements: a Mach-Zehnder modulator whose radio-frequency and dc half-wave voltages are $V_{\pi}$ and $V_{\pi_{\mathrm{dc}}}$, respectively, biased with a voltage $V_{B}$ and illuminated by a continuous-wave semiconductor laser source of power $P$, an optical fiber delay line of delay time $T_{D}$, a photodiode with gain $g$, and a radio-frequency amplifier with gain $G$ to convert the optical feedback signal into an electrical voltage $V(t)$ for the Mach-Zehnder electrode. The overall attenuation of this feedback loop (delay line, connectors, etc.) is described in terms of the parameter $A$. The electronic bandwidth of the feedback loop is supposed in first approximation to result from two cascaded linear first-order low-pass and high-pass filters, with low and high cutoff frequencies $f_{L}$ and $f_{H}$, respectively. If we consider $x(t)=\pi V(t) / 2 V_{\pi}$ as the dimensionless variable describing the system, its dynamics can be modeled by the following integro-differential delayed equation [6]:

$$
x+\tau \frac{d x}{d t}+\frac{1}{\theta} \int_{t_{0}}^{t} x(s) d s=\beta \cos ^{2}\left[x\left(t-T_{D}\right)+\phi\right],
$$

where $\beta=\pi g A G P / 2 V_{\pi}, \phi=\pi V_{B} / 2 V_{\pi_{\mathrm{dc}}}, \theta=1 / 2 \pi f_{L}$, and $\tau=1 / 2 \pi f_{H}$. Here, the three time parameters of the system have quite different orders of magnitude. For numerical simulations, we work with values compatible with those of the experimental setup, that is, $\tau=25 \mathrm{ps}, T_{D}=$ $30 \mathrm{~ns}$, and $\theta=5 \mu \mathrm{s}$.

We can now analyze the dynamics of the system with respect to the feedback strength $\beta$ (which is proportional to the feeder-laser power $P$ ) and the offset phase $\phi$ (proportional to the bias voltage of the Mach-Zehnder $V_{B}$ ). If we introduce the variable $y=\frac{1}{\tau} \int_{t_{0}}^{t} x(s) d s$, the evolution Eq. (1) may formally be rewritten as

$$
\dot{y}=x, \quad \dot{x}=-x-\epsilon y+\beta \cos ^{2}\left(x_{R}+\phi\right),
$$

where the overdot denotes the derivative relative to the dimensionless time $t / \tau$. The dynamics of the system is now

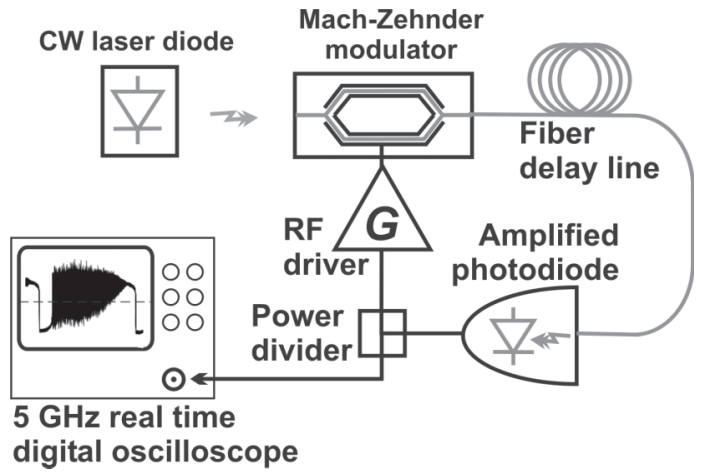

FIG. 1. The experimental setup. 
ruled by two new dimensionless parameters. The first one is the ratio between the low and the high cutoff frequencies $\epsilon=\tau / \theta=5 \times 10^{-6}$. The second one is the dimensionless delay $R=T_{D} / \tau=1.2 \times 10^{3}$, which also roughly indicates the order of magnitude for the effective number of degrees of freedom attached to the system [6]. The flow of Eq. (2) has a single stationary point $\left(x_{\mathrm{st}}, y_{\mathrm{st}}\right)=$ $\left(0, \frac{\beta}{\epsilon} \cos ^{2} \phi\right)$, and the stability of this unique fixed point can be investigated through the eigenvalue equation

$$
\lambda^{2}+\lambda+\epsilon+\beta \sin 2 \phi \lambda e^{-\lambda R}=0 .
$$

It is known that a Hopf bifurcation may occur in the system when $\lambda$ becomes pure imaginary, that is, when $\lambda=i \omega$. According to Eq. (3), the frequency $\omega$ of the limit cycle induced by the Hopf bifurcation should obey the nonlinear algebraic system

$$
\begin{gathered}
-\omega^{2}+\epsilon+\gamma \omega \sin \omega R=0, \\
1+\gamma \cos \omega R=0,
\end{gathered}
$$

where $\gamma \equiv \beta \sin 2 \phi$ is the relevant control parameter for the bifurcation. The solutions $\left(\gamma_{H}, \omega_{H}\right)$ of the above algebraic system give the critical value $\gamma_{H}$ of the control parameter for which the fixed point turns unstable and bifurcates to a limit cycle of frequency $\omega_{H}$. We can first uncouple Eqs. (4) and (5) to obtain independent equations for $\gamma$ and $\omega$ following

$$
\begin{gathered}
1+\gamma \cos \left[R \frac{\left(\gamma^{2}-1\right) \pm \sqrt{\left(\gamma^{2}-1\right)^{2}+4 \epsilon}}{2}\right]=0, \\
-\omega^{2}+\epsilon=\omega \tan \omega R,
\end{gathered}
$$

whose solutions may be approximated with an excellent precision as

$$
\gamma_{0}=-1-\epsilon R / 2, \quad \omega_{0}=\sqrt{\epsilon / R},
$$

on the one hand, and as

$$
\gamma_{k}=(-1)^{k+1}\left[1+\frac{\left(\epsilon R^{2}-k^{2} \pi^{2}\right)^{2}}{2 k^{2} \pi^{2} R^{2}}\right], \quad \omega_{k}=k \frac{\pi}{R},
$$

on the other, $k$ being a stricly positive integer.

From Eq. (5), it clearly appears that the fixed point $x=0$ is stable independently of $R$ when $|\gamma|=|\beta \sin 2 \phi|<1$. In particular, it is interesting to notice that this trivial fixed point is stable independently of $\phi$ when $\beta<1$. However, limit cycles of frequency $\omega_{k}$ may emerge when crossing the critical bifurcation value of their related counterpart $\gamma_{k}$ (which is always such that $\left|\gamma_{k}\right|>1$ ).

For positive $\gamma$, the first bifurcation occurs at $\gamma_{1}=1+$ $2.5 \times 10^{-7}$, leading to the emergence of a limit cycle of frequency $\omega_{1}=\pi / R$, corresponding to a period of $2 T_{D}=$ $60 \mathrm{~ns}$. When $\gamma$ is further increased, the fundamental frequency of that limit cycle remains constant, even though

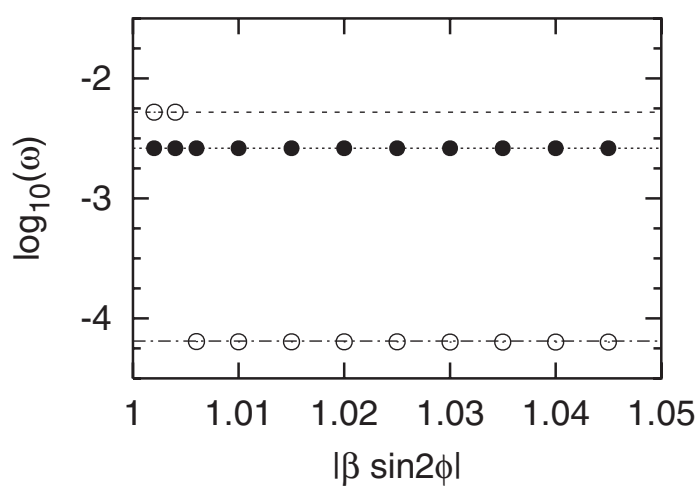

FIG. 2. Numerical variation of the limit cycles' frequencies after the Hopf bifurcations at $\gamma=-1(\bigcirc)$, and after $\gamma=1(\mathbf{O})$. The initial condition in the whole $\left[-T_{D}, 0\right]$ interval is the trivial fixed point. The three horizontal dashed lines, respectively, correspond from top to bottom to $\omega_{2}=2 \pi / R, \omega_{1}=\pi / R$, and $\omega_{0}=\sqrt{\epsilon / R}$. Note that the frequencies found numerically always perfectly coincide with one of the three aforementioned analytical frequencies.

the waveform changes from a sinusoid to a quasi-square wave [7]. Numerical simulations show that starting from an initial condition corresponding to the stationary fixed point, the system always evolves to limit cycles of period $2 T_{D}$ when $\gamma$ is increased beyond 1 (see Fig. 2), and chaos is observed when $\gamma$ is sufficiently high.

On the other hand, for $\gamma<0$, the first limit cycle to emerge appears at $\gamma_{2}=-1-9.1 \times 10^{-6}$ with a frequency $\omega_{2}=2 \pi / R$, corresponding to a period of $T_{D}=$ $30 \mathrm{~ns}$. But in this case, when $\gamma$ is increased in absolute value and reaches the value $\gamma_{0}=-1.003$, the limit cycle of frequency $\omega_{0}=\sqrt{\epsilon / R}$ emerges and becomes the
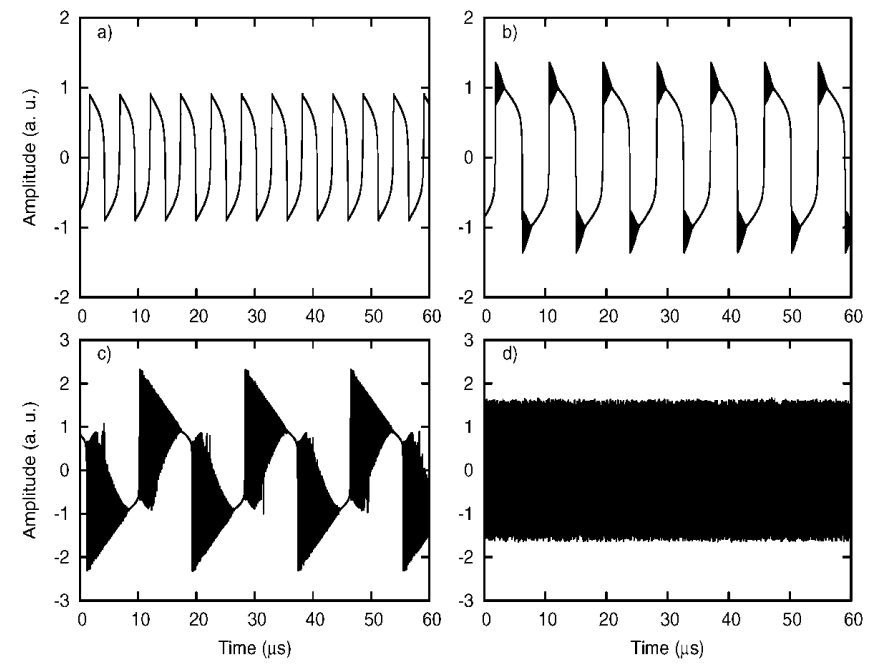

FIG. 3. Birth, evolution, and destruction of the breathers as the nonlinear feedback strength parameter $\beta$ is increased, when $\phi=$ $-\pi / 4$ (symmetric case). (a) $\beta=1.5$. (b) $\beta=2.0$. (c) $\beta=3.0$. (d) $\beta=3.5$. 

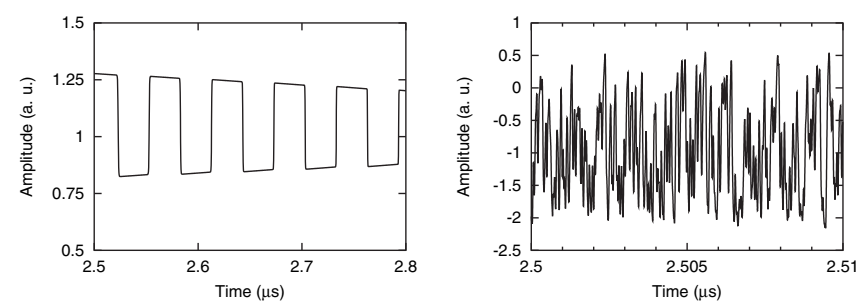

FIG. 4. Inner structure of the breathers (fast-scale dynamics). (a) Enlargement of Fig. 3(b) $(\beta=2)$ : the breathers are $2 T_{D}$ periodic. (b) Enlargement of Fig. 3(c) $(\beta=3)$ : the breathers are chaotic.

attracting limit cycle when the initial condition is taken near the unstable fixed point. Effectively, the frequency of the system's oscillations switches beyond $\gamma_{0}$ from $\omega_{2}$ to $\omega_{0}$, which is approximately 100 times smaller. Figure 2 clearly indicates this drastic change of time scale: while we have oscillations of period $T_{D}=30 \mathrm{~ns}$ when $\beta \sin 2 \phi=$ -1.001 , we rather have oscillations of period $\sim 3 \mu$ s when $\beta \sin 2 \phi=-1.01$. The emergence of a stable limit cycle whose period is significantly greater than $\tau$ and $T_{D}$ opens the way to a very interesting phenomenology.

Effectively, when $\beta$ is increased subsequently to the secondary bifurcation at $\gamma=\gamma_{0}$, we can witness the arising of a hybrid regime where a fast-scale dynamics is superimposed onto the slow-scale limit cycle. For example, it can be seen in Fig. 3(a) that when $\beta$ is increased to 1.5, the amplitude extrema begin to behave locally like paired fixed points, in the sense that the dynamics becomes significantly slow at their neighborhood. As $\beta$ is further increased, quasi-square-wave oscillations of period $2 T_{D}$ emerge at their vicinity, but as can be seen in Fig. 3(b) they are rapidly damped just after their birth, that is, as soon as the system moves away from an extremum: we refer to them as breathers, and, at this stage, these breathers are still periodic. The internal frequency and the exponential damping of the emerging breathers can also be estimated analytically. In fact, at this early stage, the mean value contribution of the feedback term $\beta \cos ^{2}\left(x_{R}+\phi\right)$ in Eq. (2) is canceled by the mean value of filter term $\epsilon y$, whose fluctuating component is negligible. Therefore, the extrema $\tilde{x}_{\text {st }}$ of the low-frequency oscillation coincide with a good approximation to the solutions of the transcendental algebraic equation $x=\frac{1}{2} \beta \cos 2(x+\phi)$. Then we can

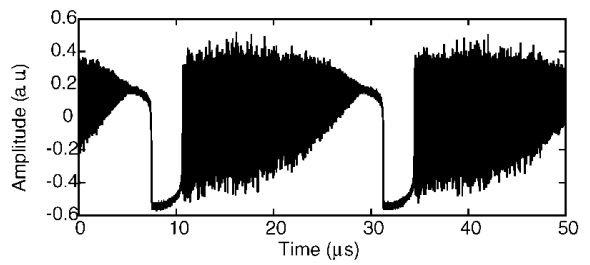

track the evolution of a perturbation $\delta \tilde{x}=\delta \tilde{x}_{0} e^{(\tilde{\kappa}+i \tilde{\omega}) t}$ around the extrema $\tilde{x}_{\mathrm{st}}$, and it is found that the breathers emerge with a winding frequency $\tilde{\omega}=\pi / R$, and are damped at a rate $\tilde{\kappa} \simeq \ln (\tilde{\gamma}) / R$, with $\tilde{\gamma}=\beta \sin 2\left(\tilde{x}_{\text {st }}+\phi\right)$. When $\beta$ is increased, the fluctuating component of the term $\epsilon y$ also increases, and it can be calculated from a multiple time scale analysis (using $\epsilon$ as the expansion parameter) that, as a consequence, the damping drops to $\tilde{\kappa} \sim-\epsilon^{-1}$; i.e., the breathers begin to span over a time scale of the order of $\theta$.

When $\beta$ continues to increase, the breathers become chaotic, as can be seen in Fig. 3(c). Here, we are in front of a hybrid regime where the chaos is triggered at a ns time scale and superimposed onto the low-frequency limit cycle whose period is of the order of a few $\mu$ s. In Fig. 3(d), it is shown that a further increase of $\beta$ leads to the destruction of the breathers and to chaotic oscillations without any trace of slow-scale dynamics. In Fig. 4, we can see the inner structure of the breathers of Fig. 3. The enlargement of Fig. 4(a) shows the quasi-square-wave $2 T_{D}$-periodic structure of the breathers of Fig. 3(b), in accordance to the winding frequency $\tilde{\omega}=\pi / R$ predicted by the theory, while Fig. 4(b) displays the fast-scale chaotic nature of the breathers shown in Fig. 3(c).

This dynamical behavior has also been recovered experimentally. In the experimental setup, the electro-optical modulator is a $10 \mathrm{GHz}$ commercial $\mathrm{LiNbO}_{3}$ integrated Mach-Zehnder modulator with $V_{\pi_{\mathrm{dc}}}=4.0 \mathrm{~V}$ and $V_{\pi}=$ $4.2 \mathrm{~V}$. The coherent optical feeder of this modulator is a distributed feedback semiconductor laser emitting at the standard infrared wavelength of $1550 \mathrm{~nm}$. The delay line is a $6 \mathrm{~m}$ long single-mode optical fiber yielding an overall time delay of $30 \mathrm{~ns}$. The wide-band amplified photodetector has a sensitivity of $2 \mathrm{~V} / \mathrm{mW}$ and a $10 \mathrm{GHz}$ bandwith. The radio-frequency electronic driver SHF100CP has a bandwidth ranging from $30 \mathrm{kHz}$ to $25 \mathrm{GHz}$, and it performs within the nonlinear feedback loop an amplification whose gain is $18 \mathrm{~dB}$ ( $26 \mathrm{dBm}$ at $1 \mathrm{~dB}$ compression). The amplitude of the background random noise of the experimental setup has been measured in the $\beta<1$ regime (small feedback gain, when the fixed point $x=0$ is a stable solution), and it is typically from 20 to 30 times smaller than the amplitude of the breathers which are obtained experimentally.

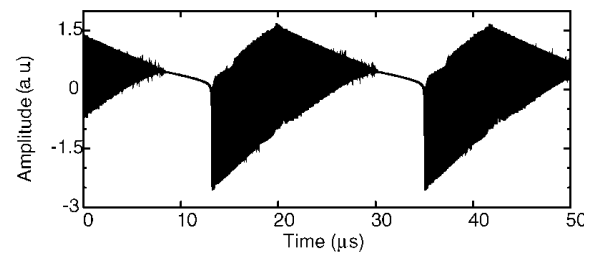

FIG. 5. Large-scale time traces of the hybrid regime of simultaneous slow-scale periodicity and fast-scale chaos. Adjacent asymmetrical breathers can also merge as they are growing. (a) Experimental time trace, with $P=4.15 \mathrm{~mW}$ and $V_{B}=3.09 \mathrm{~V}$. (b) Numerical time trace, with $\beta=2.8$ and $\phi=-\pi / 10$. 

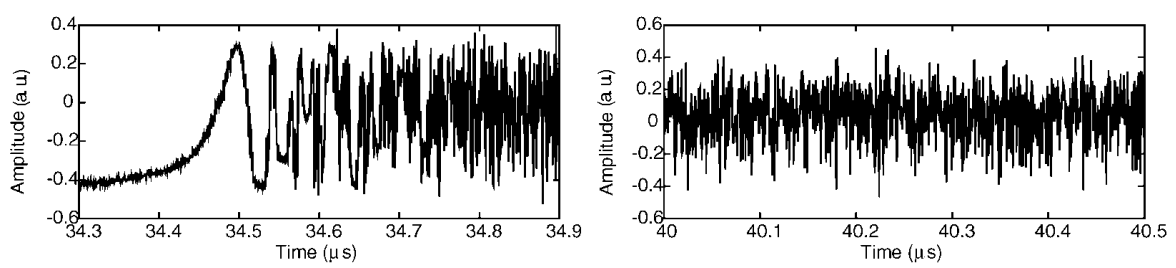

FIG. 6. Small-scale experimental time traces [enlargement of Fig. 5(a)]. (a) Onset of chaos in the breathers. Transients associated with the time delay $T_{D}$ can clearly be identified. (b) Fully developed chaos within the breathers.

Figure 5(a) presents the experimental large-scale time traces corresponding to the chaotic breathers, and Fig. 5(b) displays the related numerical simulation. These experimental breathers correspond to the most general case of asymmetric breathers (obtained when $\phi \neq-\pi / 4$ ), by opposition to the breathers of Fig. 3(c) which were symmetric relative to the horizontal axis $(\phi=-\pi / 4)$. In Fig. 6 , an enlargement of the experimental time traces is displayed, and it enables one to have a better insight into the underlying mechanism of the process. The onset of chaos at the beginning of each breather is shown in Fig. 6(a). The transients related to the time delay $T_{D}$ can clearly be recognized. Enough time is provided for these transients to evolve towards fully developed chaos as can be seen in Fig. 6(b), and therefore the occurrence of this phenomenon is not only related to the integro-differential dynamical property of the feedback loop, but also to the ratio between the different time scales of the system.

To the best of our knowledge, it is the first time that chaotic breathers are identified in a delayed dynamical system, even though it is known that chaotic breathers can exist in systems of ordinary differential equations. For example, they have been encountered in $N$-dimensional nonlinear systems with many time scales, where the nonlinearity is topologically present in a single direction of the state space: in that context, they emerged as the consequence of a nonlinear combination of $N-1$ oscillation modes, the so-called full instability regime [8]. Chaotic breathers have also been met in nonlinear systems excited by a high frequency forcing term modulated in amplitude by a slowly varying quasiperiodic envelope [9]. In our case, chaotic breathers appear as a new solution in autonomous and delayed dynamical systems, a robust and stable intermediate state between periodicity and full chaos.

In conclusion, this study has evidenced that in delayed dynamical systems, fast-scale chaos can coexist with slowscale periodicity. We have shown that this phenomenon can be observed in a relatively simple model, as the result of the interplay between a bandpass filter and a nonlinear feedback delay term. In systems where the filter is only low pass (and not bandpass) as in the Ikeda model, fastscale chaos is observed when the feedback strength is high enough; however, slow-scale periodicity (breathers) can definitively not arise. The disparity between the various time scales should also be emphasized, as in our case, for example, they span over 6 orders of magnitude. Neuronal systems also typically present a wide diversity of time scales in their dynamics, and they can display a large variety of spiking and bursting behaviors that are ruled by nonlinear evolution equations similar to the flow (2) [10]; if the propagation delay were taken into account in such models, we could expect chaotic breathers to appear. In general, depending on the ratios between their related time scales, coexistence of chaos and periodicity could also be observed in other delayed dynamical systems, provided that the delayed feedback signal is subjected to a kind of bandpass filtering.

This work has been funded by European Commission (project Occult, IST-2000-29683). Y.C.K. and P.C. acknowledge financial support from MEC (Spain) and FEDER (Grants No. FIS2004-00953, No. BFM20010341-C02-02, and No. TIC2001-4572-E). Helpful discussions with R. Herrero, G. Orriols, C. R. Mirasso, and M. Matías are acknowledged. Y.C. K. also acknowledges a research grant from the Govern de les Illes Balears.

*Corresponding author.

Electronic address: ckyanne@imedea.uib.es

[1] K. Ikeda, Opt. Commun. 30, 257 (1979).

[2] A. Neyer and E. Voges, IEEE J. Quantum Electron. 18, 2009 (1982).

[3] R. Vallée and C. Delisle, Phys. Rev. A 34, 309 (1986).

[4] R. Vallée, P. Dubois, M. Côté, and C. Delisle, Phys. Rev. A 36, 1327 (1987); R. Vallée and C. Mariott, Phys. Rev. A 39, 197 (1989).

[5] F. A. Narducci, D. W. Bromley, G.-L. Oppo, and J. R. Tredicce, Opt. Commun. 75, 184 (1990).

[6] J.-P. Goedgebuer, P. Levy, L. Larger, C.-C. Chen, and W. T. Rhodes, IEEE J. Quantum Electron. 38, 1178 (2002); N. Gastaud, S. Poinsot, L. Larger, J.-M. Merolla, M. Hanna, J.-P. Goedgebuer, and F. Malassenet, Electron. Lett. 40, 898 (2004).

[7] T. Erneux, L. Larger, M. W. Lee, and J.-P. Goedgebuer, Physica (Amsterdam) 194D, 49 (2004).

[8] J. Rius et al., Phys. Rev. E 62, 333 (2000); Chaos10, 760 (2000).

[9] Z. Qu, G. Hu, G. Yang, and G. Qin, Phys. Rev. Lett. 74, 1736 (1995).

[10] E. M. Izhikevich, Int. J. Bifurcation Chaos Appl. Sci. Eng. 10, 1171 (2000), and references therein. 